\title{
CONTROL GENETICO DE LA RESISTENCIA Y DEL ESCAPE A Teratosphaeria nubilosa EN Eucalyptus globulus EN URUGUAY
}

\author{
Balmelli, Gustavo ${ }^{3}$; Simeto, Sofía ${ }^{3}$; Torres-Dini, Diego ${ }^{3}$; Castillo, Alicia $^{3}$; \\ Altier, Nora ${ }^{3}$; Mac Gregor, Juan ${ }^{4}$ y Diez, Julio ${ }^{5}$
}

\section{RESUMEN}

El Eucalyptus globulus es la especie forestal más plantada en el Uruguay, existiendo más de 270.000 hectáreas de plantaciones comerciales. Sin embargo actualmente está siendo sustituido por otras especies, como E. grandis y E. dunnii, debido a los severos daños foliares provocados por Teratosphaeria nubilosa en las plantaciones jóvenes.

La continuidad en la plantación de esta especie depende del rápido desarrollo de material genético resistente a la enfermedad. La variación genética de la resistencia del follaje juvenil y la velocidad del cambio de follaje de juvenil a adulto fueron evaluadas en una prueba de progenies de E. globulus naturalmente infectada por T. nubilosa. El material genético incluyó 194 familias de polinización abierta provenientes de dos huertos semilleros pertenecientes al Instituto Nacional de Investigación Agropecuaria (INIA).

A los 14 meses de edad se evaluó la severidad del daño (\% del área foliar afectada con manchas y \% de defoliación) y la precocidad del cambio de follaje (\% de follaje adulto). La severidad del daño presentó moderada heredabilidad individual $\left(\mathrm{h}^{2}=0.46 \pm 0.06\right.$ para el área foliar manchada y $0.34 \pm 0.05$ para la defoliación), pero una muy baja varianza genética aditiva $\left(\sigma^{2}{ }_{\mathrm{A}}=\right.$ $6.25 \pm 0.90$ para manchas y $29.55 \pm 5.21$ para defoliación). En cambio, el porcentaje de follaje adulto presentó una alta heredabilidad $\left(\mathrm{h}^{2}=0.62 \pm 0.06\right)$ y una alta varianza genética aditiva $\left(\sigma_{\mathrm{A}}^{2}=\right.$ $155.85 \pm 19.29)$. La correlación genética entre el porcentaje de manchas y el porcentaje de defoliación fue positiva $\left(r_{G}=0.48 \pm 0.09\right)$ mientras que la correlación genética entre la severidad del daño (porcentaje de manchas y porcentaje de defoliación) y la precocidad del cambio de follaje fue negativa $\left(r_{G}=-0.34 \pm 0.09\right.$ y $-0.74 \pm 0.06$, respectivamente).

Los resultados obtenidos sugieren que las posibilidades de selección en E. globulus por resistencia del follaje juvenil a $T$. nubilosa son muy limitadas y que en cambio existen buenas posibilidades de seleccionar por precocidad en el cambio de follaje. Se concluye que la selección en base al cambio precoz de follaje, es decir por escape a la enfermedad, es la estrategia más adecuada para obtener material genético de E. globulus apto para zonas con alto riesgo de infección de T. nubilosa.

Palabras clave: Control genético, Eucalyptus globulus, escape, resistencia, Teratosphaeria nubilosa.

\footnotetext{
${ }^{3}$ Programa Nacional Forestal. Instituto Nacional de Investigación Agropecuaria (INIA). Ruta 5, Km 386.

Tacuarembó, Uruguay. gbalmelli@tb.inia.org.uy

${ }^{4}$ Oficina Técnica. Sierras Calmas. Mariscal Estigarribia 928. Montevideo, Uruguay.

${ }^{5}$ Departamento de Producción Vegetal y Recursos Forestales. Instituto de Gestión Forestal Sostenible. Universidad de Valladolid. Avenida Madrid 44. Palencia, España.
} 


\section{SUMMARY}

Eucalyptus globulus is the most widely planted species in Uruguay accounting for more than 270.000 ha of commercial planted forests. However, currently the species is replaced by other species, as Eucalyptus grandis and E. dunnii, because of the severe foliage damages to the first caused by Teratosphaeria nubilosa in young planted forests.

Future of $E$. globulus plantations depends on resistant genetic material development. Young foliage resistance genetic variation and its speed to change to mature foliage were evaluated in an E. globulus naturally infested with $T$. nubilosa progenies test, including 194 open pollinated families from the Instituto Nacional de Investigación Agropecuaria (INIA) seed orchard.

Damage severity (foliage area affected percent and defoliation percent) and foliage change precocity (mature leaves percent) at 14 months old were evaluated. Damage severity presented a moderate individual heritability $\left(\mathrm{h}^{2}=0.46 \pm 0.06\right.$ for foliage area and $0.34 \pm 0.05$ for defoliation), but a very low genetic additive variance $\left(\sigma^{2}{ }_{A}=6.25 \pm 0.90\right.$ for foliage area y $29.55 \pm$ 5.21 for defoliation).

Regarding to the mature foliage percent, it presented a high heritability $\left(h^{2}=0.62 \pm 0.06\right)$ and a high genetic additive variance $\left(\sigma_{\mathrm{A}}^{2}=155.85 \pm 19.29\right)$. Genetic correlation between foliage patches and defoliation percent was positive $\left(r_{G}=0.48 \pm 0.09\right)$ while genetic correlation between damage severity (patches percent and defoliation percent) and foliage change precocity was negative ( $r_{G}=-0.34 \pm 0.09$ and $-0.74 \pm 0.06$, respectively).

Obtained results suggest that selection possibilities in $E$. globulus by young foliage resistance to $T$. nubilosa are low, while there are good selection possibilities by foliage change precocity. It is concluded that by precoty in foliage change (disease escape) is the most appropriate strategy to obtain $E$. globulus genetic material suitable to zones with high $T$. nubilosa infection risk.

Key words: Genetic control, Eucalyptus globulus, escape, resistance, Teratosphaeria nubilosa. 


\section{INTRODUCCION}

El Eucalyptus globulus es la especie forestal más plantada en el Uruguay, existiendo más de 270.000 hectáreas de plantaciones comerciales (MGAP 2012). Sin embargo, la falta de adaptación al clima y la alta susceptibilidad de la especie a diferentes enfermedades han restringido su plantación a la zona SE del país, la de mejor aptitud para E. globulus.

En el año 2007 se registra por primera vez en Uruguay la presencia de Teratosphaeria nubilosa (Pérez et al. 2009), patógeno que provoca la enfermedad conocida comúnmente como Manchas de Mycosphaerella. E. globulus es altamente susceptible a esta enfermedad, la cual provoca manchas foliares y defoliación en plantaciones jóvenes, previo al cambio de follaje (Carnegie et al. 1998; Hunter et al. 2009).

Relevamientos sistemáticos conducidos desde el año 2008 en plantaciones de eucaliptos en Uruguay han demostrado que la enfermedad está distribuida en todo el país y causa defoliación severa en E. globulus (Balmelli et al. 2009; Balmelli et al. 2011; Simeto et al. 2010). El nivel de daño que año a año viene generando este patógeno en plantaciones de uno y dos años está provocando la sustitución de esta especie por otras más resistentes, como E. grandis y E. dunnii.

Dada la situación descrita, la continuidad de la plantación de E. globulus en el país depende del rápido desarrollo de material genético resistente a la enfermedad.

Con el fin de analizar las posibilidades de selección de materiales de buen comportamiento frente a la enfermedad se evaluó una prueba de progenies de E. globulus naturalmente infectada por $T$. nubilosa. Los objetivos del estudio fueron estimar la variación genética de la resistencia del follaje juvenil y de la velocidad del cambio de follaje de juvenil a adulto y estimar las ganancias genéticas que podrían obtenerse mediante diferentes estrategias de selección.

\section{MATERIALES Y METODOS}

La susceptibilidad a $T$. nubilosa y la precocidad del cambio de follaje fueron evaluados en una prueba de progenies de E. globulus instalada en marzo de 2011 en el departamento de Lavalleja (341ํ' LS; 54ำ5' LO; 206 msnm). El material genético incluyó 194 familias de polinización abierta provenientes de dos huertos semilleros pertenecientes al Instituto Nacional de Investigación Agropecuaria (INIA). El diseño experimental fue de bloques completos al azar, con 3 repeticiones y parcelas de 8 plantas en línea. La densidad de plantación fue de 1.428 plantas por hectárea (3,5 metros entre líneas y 2 metros sobre líneas).

En febrero de 2012 se registraron varios días consecutivos de lluvia y/o alta humedad relativa, lo cual desencadenó una severa infección de $T$. nubilosa. La severidad del daño foliar y la precocidad del cambio de follaje fueron evaluados en mayo de 2012, a los 14 meses de edad. La susceptibilidad a la enfermedad fue cuantificada en cada árbol utilizando dos parámetros; la severidad de las manchas foliares (porcentaje del área foliar con necrosis) y la defoliación (porcentaje de hojas caídas). En ambos casos se utilizaron escalas visuales adaptadas de Lundquist y Purnell (1987) y de Carnegie et al. (1994). Para la evaluación de la severidad de manchas (SEV) cada árbol se caracterizó de acuerdo a las siguientes clases de daño: 0, 5, 10, 15, $20,25,30,35$ y $40 \%$, mientras que las clases utilizadas para la evaluación de la defoliación (DEF) fueron: $0,10,20,30,40,50,60$ y 70\%. Para evaluar la precocidad del cambio de follaje se midió la proporción de follaje adulto (ADFO), utilizando las siguientes clases: $0,10,20,30,40,50,60,70$, 80 y $90 \%$. 
Para cada característica evaluada se estimaron los componentes de varianza mediante un modelo mixto en ASReml (Version 3.0) (Gilmour et al. 2009). El modelo univariado utilizado fue:

$$
\begin{aligned}
& \mathrm{Y}=\mathrm{MU}+\mathrm{REP}+F A M+R E P^{*} F A M+E R R O R \\
& \sigma_{\mathrm{p}}^{2}=\sigma_{\mathrm{f}}^{2}+\sigma_{\mathrm{rf}}^{2}+\sigma_{\mathrm{e}}^{2} \\
& \sigma_{\mathrm{a}}^{2}=\frac{\sigma_{\mathrm{f}}^{2}}{\mathrm{r}} \\
& h_{\mathrm{op}}^{2}=\frac{\sigma_{\mathrm{a}}^{2}}{\sigma_{0}^{2}}
\end{aligned}
$$

REP es el efecto fijo de la repetición

FAM es el efecto aleatorio de la familia

$R E P^{*} F A M$ es la interacción de repetición por familia

La varianza fenotípica $\left(\sigma_{\mathrm{p}}^{2}\right)$, la varianza aditiva $\left(\sigma_{\mathrm{a}}^{2}\right)$ y la heredabilidad individual $\left(h_{\mathrm{op}}^{2}\right)$ fueron calculadas como:

Donde: $\quad \sigma_{\mathrm{f}}^{2}$ es la varianza de familias

$\sigma_{\mathrm{rf}}^{2}$ es la varianza de la interacción repetición por familia, $\sigma_{\mathrm{e}}^{2}$ es la varianza residual

$r$ es el coeficiente de parentesco, fijado en 0.4 para ajustar la desviación respecto a la relación de medios hermanos (asumiendo un 30\% de autofecundación) (Volker et al. 1990).

Análisis bivariados fueron también implementados en ASReml, ajustando el mismo modelo que en los análisis univariados y utilizando los componentes de varianza de dichos análisis como valores de inicio. Las correlaciones genéticas entre diferentes características $\left(r_{a}\left(i_{i}, j\right)\right.$ fueron calculadas con las varianzas y covarianzas aditivas como:

$$
r_{\mathrm{a}(\mathrm{i}, j \mathrm{j})}=\frac{\sigma_{\mathrm{a}(\mathrm{i}, j)}}{\sqrt{\sigma_{\mathrm{a}(i)}^{2} \sigma_{\mathrm{a}(j)}^{2}}}
$$

Donde: $\quad \sigma_{\mathrm{a}(\mathrm{d}) \text { y }}^{2} \sigma_{\mathrm{a}(\hat{j})}^{2}$ son las varianzas aditivas para las características $i$ y $j$, respectivamente

$\sigma_{a(i, j)}$ es la covarianza aditiva entre ambas características.

Las ganancias genéticas esperadas mediante selección por resistencia y mediante selección por precocidad en el cambio de follaje fueron analizadas para dos estrategias; la transformación de la prueba de progenies en un huerto semillero y la selección y clonación de individuos. Para el primer caso se simuló la retención de las mejores 30 familias y del mejor 
individuo de cada parcela, mientras que para el segundo caso se simuló la selección de los mejores 200 individuos.

Para la estimación de las ganancias genéticas $(\Delta G)$ esperadas, expresadas en porcentaje, se utilizaró los valores de cría parentales e individuales obtenidos para las diferentes características en los análisis univariados.

\section{RESULTADOS Y DISCUSION}

Al momento de realizar la evaluación del ensayo, tres meses después de que se dieran condiciones de infección, la severidad del daño provocado por $T$. nubilosa fue en promedio de $10,5 \%$ para el área foliar manchada (con un rango a nivel de individuos entre 0 y $40 \%$ ) y de $31,5 \%$ para la defoliación (con un rango entre 0 y $70 \%$ ), mientras que el valor medio para follaje adulto fue de $9,9 \%$ (con un rango entre 0 y $90 \%$ ). La baja proporción de follaje adulto se explica por lo temprano de la evaluación (14 meses), ya que el cambio de follaje en $E$. globulus comienza generalmente entre los 18 y los 24 meses. De todas formas la severidad del daño, tanto para el área foliar manchada como para la defoliación, presentó una variabilidad mucho menor que la presentada por la precocidad del cambio de follaje (Cuaro 1). La escasa variabilidad observada en el nivel de daño probablemente se deba a la alta susceptibilidad que presenta $E$. globulus a la enfermedad (Carnegie et al. 1998). A su vez, la heredabilidad individual para la severidad del daño fue moderada $\left(\mathrm{h}^{2}=0,46 \pm 0,06\right.$ para el área foliar manchada y $0,34 \pm 0,05$ para la defoliación) y fue alta para el porcentaje de follaje adulto $\left(h^{2}=0,62 \pm 0,06\right)$ (Cuadro $\left.N^{\circ} 1\right)$.

\section{Cuadro $\mathrm{N}^{\circ} 1$ \\ VARIANZA FENOTÍPICA, VARIANZA ADITIVA Y HEREDABILIDAD INDIVIDUAL A LOS 14 MESES EN PRUEBA DE PROGENIES DE E. globulus EN LAVALLEJA (URUGUAY).}

\begin{tabular}{|c|c|c|c|c|}
\hline Característica & Media & $\begin{array}{c}\text { Varianza } \\
\text { Fenotípica } \\
\sigma_{\mathrm{p}}^{2} \pm \text { ee }\end{array}$ & $\begin{array}{l}\text { Varianza } \\
\text { Aditiva } \\
\sigma_{\mathrm{a}}^{2} \pm \mathrm{ee}\end{array}$ & $\begin{array}{l}\text { Heredabilidad } \\
\text { Individual } \\
h_{\mathrm{op}}^{2} \pm \text { ee }\end{array}$ \\
\hline SEV (\%) & 10,5 & $13,67 \pm 0.43$ & $6,25 \pm 0,90$ & $0,46 \pm 0,06$ \\
\hline DEF (\%) & 31,5 & $86,16 \pm 2.63$ & $29,55 \pm 5,21$ & $0,34 \pm 0,05$ \\
\hline ADFO (\%) & 9,9 & $251,44 \pm 8.70$ & $155,85 \pm 19,29$ & $0,62 \pm 0,06$ \\
\hline $\begin{array}{ll}\text { ee } & \text { : errores } \\
\text { SEV } & \text { : porcenta } \\
\text { DEF } & \text { : defoliaci } \\
\text { ADFO } & \text { : porcenta }\end{array}$ & $\begin{array}{l}\text { (ee) } \\
\text { ea folia } \\
\text { laje adı }\end{array}$ & ada & & \\
\hline
\end{tabular}

Los valores de heredabilidad obtenidos para la susceptibilidad a $T$. nubilosa se encuentran dentro del rango de valores reportados por otros autores para E. globulus. Potts et al. (2004) reportan una heredabilidad de 0,60 para la severidad de T. nubilosa; Reinoso (1992) entre 0,23 y 0,48 para la severidad del daño de Mycosphaerella; Dungey et al. (1997) entre 0,2 y 0,21 para el daño de $T$. nubilosa y $T$. cryptica y Carnegie y Ades (2005) entre 0,17 y 0,36 para el daño de $T$. cryptica. 
La heredabilidad obtenida para el porcentaje de follaje adulto también es similar a la obtenida por otros autores para la precocidad del cambio de follaje en E. globulus, por ejemplo López et al. (2002) reportan heredabilidades entre 0,54 y 0,65; Jordan et al. (1999) entre 0,43 y 0,74 y Hamilton et al. (2011) entre 0,44 y 0,65.

Estos resultados sugieren que es posible seleccionar tanto por resistencia a $T$. nubilosa como por precocidad en el cambio de follaje, es decir por escape a la enfermedad.

Sin embargo, la varianza total, la varianza aditiva y la heredabilidad para la precocidad en el cambio de follaje son mayores que para la resistencia, lo cual indica que la respuesta a la selección por escape a la enfermedad sería mayor.

Por otra parte, debido a que la evaluación de daño se realizó en el total de la copa, en aquellos árboles que presentaban follaje adulto (el cual presenta manchas pero que no se cae) la resistencia a la defoliación puede estar sobrestimada.

Cuando se analiza la defoliación utilizando el \% de follaje adulto como covariable, tanto la varianza aditiva $(14,33 \pm 3,44)$ como la heredabilidad $(0,21 \pm 0,05)$ presentan valores mucho menores que los presentados en el Cuadro $\mathrm{N}^{\circ} 1$.

Esto demuestra que la variabilidad genética real (per se) de la resistencia a la defoliación es aún menor que la estimada inicialmente, lo cual limita aún más las posibilidades de selección.

Las correlaciones fenotípicas y genéticas entre el porcentaje de manchas y el porcentaje de defoliación fueron positivas (Cuadro $N^{\circ} 2$ ), lo cual es esperable dado que ambas características son diferentes síntomas de la misma enfermedad.

Por el contrario, las correlaciones fenotípicas y genéticas entre la severidad del daño (porcentaje de manchas y porcentaje de defoliación) y la precocidad del cambio de follaje fueron negativas (Cuadro $\mathrm{N}^{\circ} 2$ ). En otras palabras, cuanto mayor es el porcentaje de follaje adulto menor es el nivel de daño provocado por la enfermedad.

Hay que considerar sin embargo que, como se mencionó anteriormente, la defoliación covaría con el porcentaje de follaje adulto, por lo que la correlación entre ambas características está sobreestimada.

Cuadro $\mathrm{N}^{\circ} 2$

CORRELACIONES FENOTÍPICAS Y GENÉTICAS A LOS 14 MESES EN PRUEBA DE PROGENIES DE E. globulus EN LAVALLEJA (URUGUAY)

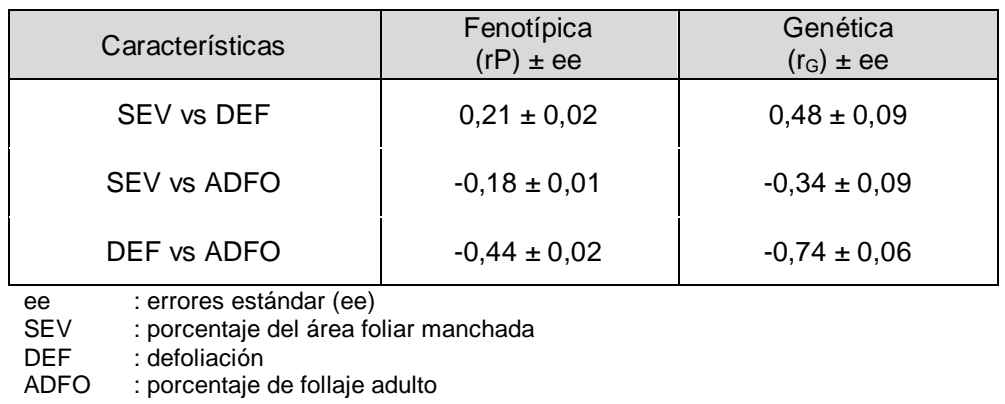


Las ganancias genéticas esperadas mediante la conversión de la prueba de progenies en un huerto semillero son relativamente bajas cuando se selecciona por bajo nivel de manchas y bajo nivel de defoliación (Cuadro $\mathrm{N}^{\circ} 3$ ).

Por el contrario, cuando la selección se realiza por porcentaje de follaje adulto se esperan muy altas ganancias genéticas. Similares resultados se esperan mediante la selección y clonación de los mejores individuos (Cuadro $N^{\circ} 3$ ).

\section{Cuadro $\mathrm{N}^{\circ} 3$}

GANANCIAS GENÉTICAS ESPERADAS PARA SEVERIDAD DE MANCHAS, DEFOLIACIÓN Y PORCENTAJE DE FOLLAJE ADULTO MEDIANTE LA TRANSFORM ACIÓN DE LA PRUEBA DE PROGENIES (PP) EN UN HUERTO SEMILLERO (HS) Y MEDIANTE LA SELECCIÓN Y CLONACIÓN DE INDIVIDUOS

\begin{tabular}{|c|c|c|c|c|c|c|c|}
\hline \multirow[b]{2}{*}{$\begin{array}{l}\text { Criterio } \\
\text { Selección }\end{array}$} & \multirow[b]{2}{*}{$\begin{array}{l}\text { Media } \\
\text { Actual }\end{array}$} & \multicolumn{3}{|c|}{ Transformación de PP en HS } & \multicolumn{3}{|c|}{ Selección y Clonación de individuos } \\
\hline & & $\begin{array}{l}\text { Media } \\
\text { Pob. } \\
\text { Selecta }\end{array}$ & $\begin{array}{l}\text { Diferencial } \\
\text { Selección }\end{array}$ & $\begin{array}{c}\text { Ganancia } \\
\text { Genética } \\
\text { Esperada } \\
\Delta \mathrm{G}\end{array}$ & $\begin{array}{l}\text { Media } \\
\text { Pob. } \\
\text { Selecta }\end{array}$ & $\begin{array}{l}\text { Diferencial } \\
\text { Selección }\end{array}$ & $\begin{array}{c}\text { Ganancia } \\
\text { Genética } \\
\text { Esperada } \\
\Delta \mathrm{G}\end{array}$ \\
\hline SEV (\%) & 10.5 & 5.7 & 4.8 & 21.2 & 4.8 & 5.7 & 25.0 \\
\hline DEF (\%) & 31.5 & 14.3 & 17.2 & 18.6 & 10.1 & 21.4 & 23.1 \\
\hline ADFO (\%) & 9.9 & 51.4 & 41.5 & 258.6 & 51.6 & 41.7 & 259.9 \\
\hline $\begin{array}{l}\text { SEV } \\
\text { DEF } \\
\text { ADFO }\end{array}$ & ación & & chada & & & & \\
\hline
\end{tabular}

Los resultados obtenidos sugieren que las posibilidades de selección en E. globulus por resistencia del follaje juvenil a $T$. nubilosa son muy limitadas. A su vez, desde el punto de vista operativo, esta estrategia tiene la desventaja de que la evaluación de germoplasma depende de la ocurrencia de condiciones ambientales favorables para la infección. En cambio, la selección por precocidad en el cambio de follaje no solo puede realizarse en forma segura (con independencia de las condiciones ambientales) sino que además podría utilizarse en forma preventiva en países como Chile, donde este patógeno aún no ha ingresado.

Se concluye que la selección en base al cambio precoz de follaje, es decir por escape a la enfermedad, es la estrategia más adecuada para obtener material genético de $E$. globulus para zonas con riesgo de infección de $T$. nubilosa.

\section{RECONOCIMIENTOS}

A la empresa Sierras Calmas por proveer el sitio, por la preparación del terreno y por el mantenimiento del ensayo. Al personal de apoyo del Programa Forestal del INIA, Pablo Núñez, Federico Rodríguez, Wilfredo González y Marcelo Alfonso, por su colaboración en la preparación de las plantas, la instalación del ensayo y la evaluación del mismo. El estudio fue parcialmente financiado por una beca otorgada al primer autor por el Instituto Nacional de Investigación y 
Tecnología Agraria y Alimentaria (INIA) de España, en el marco del Programa de Formación del Sistema de los INIA de Iberoamérica.

\section{REFERENCIAS}

Balmelli, G.; Martínez, G.; Simeto, S.; Torres, D.; Pérez, C.A.; Fros, D. y Bentancur, O., 2009. Daño foliar causado por enfermedades y plagas en plantaciones jóvenes de Eucalyptus globulus en Uruguay. Poster en XIII Congreso Forestal Mundial. 18-23 Octubre. Buenos Aires, Argentina.

Balmelli, G.; Simeto, S.; Martínez, G.; Torres, D.; Pérez, C.A. and Bentancur, O., 2011. Incidence and severity of pests and diseases on young plantations of Eucalyptus globulus in Uruguay. En: IUFRO Forest Protection Joint Meeting: pathogens, insects and their associations affecting forestry worldwide. 8-11 Noviembre. Colonia del Sacramento, Uruguay.

Carnegie, A. J. and Ades, P. K., 2005. Variation in Eucalyptus globulus Labill. and E. nitens Dean and Maiden in susceptibility of adult foliage to disease caused by Mycosphaerella cryptica (Cooke) Hansf. Silvae Genetica. 54, 174-184.

Carnegie, A. J.; Keane, P. J.; Ades, P. K. and Smith, I. W., 1994. Variation in susceptibility of Eucalyptus globulus provenances to Mycosphaerella leaf disease. Canadian Journal of Forest Research. 24, 1751-1757.

Carnegie, A. J.; Ades, P. K.; Keane, P. J. and Smith, I. W., 1998. Mycosphaerella disease of juvenile foliage in a Eucalypts species and provenance trial in Victoria, Australia. Australian Forest. 61, 190-194.

Dungey, H. S.; Potts, B. M.; Carnegie, A. J. and Ades, P. K., 1997. Mycosphaerella leaf disease - genetic variation in damage to Eucalyptus nitens, Eucalyptus globulus, and their F-1 hybrid. Canadian Journal of Forest Research. 27, 750-759.

Gilmour, A. R.; Gogel, B. J.; Cullis, B. R. and Thompson, R., 2009. ASReml User Guide Release 3.0. VSN International Ltd: Hemel Hempstead, UK.

Hamilton, M. G.; Tilyard, P. A.; Williams, D. R.; Vaillancourt, R. E.; Wardlaw, T. J. and Potts, B. M., 2011. The genetic variation in the timing of heteroblastic transition in Eucalyptus globulus is stable across environments. Australian Journal of Botany. 59, 170-175.

Hunter, G. C.; Crous, P. W.; Carnegie, A. J. and Wingfield, M. J., 2009. Teratosphaeria nubilosa, a serious leaf disease pathogen of Eucalyptus spp. in native and introduced areas. Molecular Plant Pathology. 10, 1-14.

Jordan, G. J.; Potts, B. M. and Wiltshire, R., 1999. Strong, independent quantitative genetic control of vegetative phase change and first flowering in Eucalyptus globulus ssp. globulus (Tasmanian Blue Gum). Heredity $83,179-187$.

Lopez, G. A.; Potts, B. M.; Dutkowski, G. W.; Apiolaza, L. A. and Gelid, P .E., 2002. Genetic variation and inter-trait correlations in Eucalyptus globulus base population trials in Argentina. Forest Genetics. 9, 217-231.

Lundquist, J. E. and Purnell, R. C., 1987. Effects of Mycosphaerella leaf spot on growth of Eucalyptus nitens. Plant Disease. 71, 1025-1029.

MGAP, 2012. Recurso Forestal: bosques plantados registrados. http://www.mgap.gub.uy Acceso 01/03/2012.

Pérez, G.; Hunter, G.; Slippers, B.; Pérez, C. A.; Wingfield, B. D. and Wingfield, M. J., 2009. Teratosphaeria (Mycosphaerella) nubilosa, the causal agent of Mycosphaerella leaf disease (MLD), recently introduced into Uruguay. European Journal of Plant Pathology. 125, 109-118.

Potts, B. M.; Milgate, A.; Joyce, K.; Mohammed, C.; Vaillancourt, R. E. and Dutkowski, G. W., 2004. Quantitative genetic control of Mycosphaerella resistance in Eucalyptus globulus and impact on growth. In: Eucalyptus in a Changing World. Proceedings of IUFRO conference. Aveiro, Portugal. (Ed. by N. Borralho, J. S. Pereira, C. Marques, J. Coutinho, M. Madeira, M. Tomé. pp. 46-61. 
Reinoso, C., 1992. Variation in Eucalyptus globulus in susceptibility to Mycosphaerella leaf diseases. Master of Forest Science thesis, University of Melbourne, Australia.

Simeto, S.; Balmelli, G.; Martínez, G.; Torres, D.; González, P.; Fros, D.; Bentancur, O. y Pérez, C. A., 2010. Relevamiento sanitario de Eucalyptus spp. en el Sureste y Litoral Oeste del país. Tacuarembó, Uruguay. INIA Serie Actividades de Difusión 629.

Volker, P. W.; Dean, C. A.; Tibbits, W. N. and Ravenwood, I. C., 1990. Genetic parameters and gains expected from selection in Eucalyptus globulus in Tasmania. Silvae Genetica. 39, 18-21. 
